4. Nikolopoulos D, Fanouriakis A, Boumpas DT. Update on the pathogenesis of central nervous system lupus. Curr Opin Rheumatol 2019;31(6):669-77.

5. Schwartz N, Stock AD, Putterman C. Neuropsychiatric lupus: new mechanistic insights and future treatment directions. Nat Rev Rheumatol 2019;15(3):137-52.

\section{COVID-19 AND SLE - WHAT DO WE KNOW TODAY?}

Fabrizio Conti. Sapienza University of Rome, Italy

10.1136/lupus-2020-la.21

Six months following the beginning of Covid-19 pandemic in China, data on the risk of SARS-CoV-2 infection among patients with autoimmune rheumatic diseases are now available. However, the rapid spread of the pandemic has not allowed proper design of prospective studies, thus evidence came mostly from case series and observational studies.

The early enthusiasm on hydroxychloroquine (HCQ) antiviral properties should not suggest that patients who are longterm treated with antimalarials, such as patients with systemic lupus erythematosus (SLE), are protected against SARS-CoV-2 infection. Indeed, a French report on 17 HCQ-treated SLE patients dampened the enthusiasm. ${ }^{1}$

A recent report from Covid-19 Global Rheumatology Alliance has described 80 SLE patients with Covid-19, mostly females under 65 years of age, 64\% of whom were already taking HCQ before the infection: the rate of hospitalisation and the need for intensive care did not differ between patients who were and those who were not taking HCQ. ${ }^{2}$ A study group from Northern Italy - the Italian epicentre of the pandemic - reported an incidence of 2.5\% of Covid-19 (higher compared to the general population of the same region) in 165 patients with SLE. ${ }^{3}$

Patients with SLE are possibly at risk of developing symptomatic or severe Covid-19, not only because of their disease or treatment but as a consequence of associated comorbidities known to worsen the outcome of SARS-COv-2 infection. ${ }^{4} 5$

What do we know so far? SLE patients should not withdraw their medication. Before drawing any other conclusion, large registry data are needed to clarify the incidence and the outcome of Covid-19 in patients with SLE.

\section{Learning Objectives}

- Describe the current evidence for risk of SARS-CoV-2 infection among patients with autoimmune rheumatic

diseases, notably SLE

- Explain why it is important to ensure robust evidence are available to clarify the outcome of Covid-19 in patients with SLE

\section{REFERENCES}

1. Mathian A, Mahevas M, Rohmer J, et al. Clinical course of coronavirus disease 2019 (COVID-19) in a series of 17 patients with systemic lupus erythematosus under long-term treatment with hydroxychloroquine. Ann Rheum Dis 2020;79 (6):837-39.

2. Konig MF, Kim AH, Scheetz MH, et al. Baseline use of hydroxychloroquine in systemic lupus erythematosus does not preclude SARS-CoV-2 infection and severe COVID-19. Ann Rheum Dis 2020 doi: 10.1136/annrheumdis-2020-217690 [published Online First: 2020/05/10].

3. Bozzalla Cassione E, Zanframundo G, Biglia A, et al. COVID-19 infection in a northern-Italian cohort of systemic lupus erythematosus assessed by telemedicine. Ann Rheum Dis 2020 doi: 10.1136/annrheumdis-2020-217717 [published Online First: 2020/05/14].

4. Wallace $B$, Waher $L$, Correspondence regarding Research Letter to the Editor by Mathian A, et al. 'Clinical course of coronavirus disease 2019 (COVID-19) in a series of 17 patients with systemic lupus under long-term treatment with hydroxychloroquine'. Ann Rheum Dis 2020. doi:10.1136/annrheumdis-2020-217794.

5. Gianfrancesco M, Hyrich KL, Al-Adely $\mathrm{S}$, et al. Characteristics associated with hospitalisation for COVID-19 in people with rheumatic disease: data from the COVID19 Global Rheumatology Alliance physician-reported registry. Ann Rheum Dis 2020;79(7):859-66.

\section{Meet the Editor}

\section{WHAT IS HAPPENING IN THE WORLD OF PUBLISHING?}

Ronald van Vollenhoven. Amsterdam University Medical Centers, The Netherlands

\subsection{6/lupus-2020-la.22}

In this three-part workshop we will discuss the big changes taking place in the world of publishing, how successfully to submit your next paper, and what it takes to be a great reviewer.

The world of medical-scientific publishing is undergoing dramatic change at a rapid pace. The traditional model of printed journals, to which individuals and institutions can subscribe, has been upended by the emergence of open-access journals, whose publications are accessible online for all. Traditional journals have relatively high costs associated with producing and distributing printed materials to their readers, and they derive their income from subscriptions, institutions paying the lion's share. The business model for online journals is based in part on much reduced costs, and on charging authors for publishing. Advertising features prominently in both models. At the start of the third decennium, it is clear that openaccess has been embraced by political and societal forces. The biggest drawbacks are the complexity of having two systems side by side, shifting costs to scientists without compensation, and the proliferation of non-serious, 'predatory' on-line publications.

Publishing remains one of the main obligations and challenges for the aspiring scientist. In this workshop, I will discuss 'tips and tricks' for the process of submitting and revising your manuscript.

Reviewing the work of fellow scientists is an honor and a credit to your standing as a scientist. I will discuss how you can make reviewing papers enjoyable, stimulating and a win-win. A good review should be factual, scholarly, generous, and concise: 'brevity is the soul of wit' (Hamlet, William Shakespeare).

\section{Roundtable: Refractory Lupus Manifestations: Definition and Treatment}

\section{\begin{tabular}{|l|l}
23 & REFRACTORY LUPUS NEPHRITIS
\end{tabular}}

Sandra Navarra. University of Santo Tomas, Manila, Philippines

\subsection{6/lupus-2020-la.23}

Lupus nephritis (LN) affects approximately 50-70\% and accounts for the highest morbidity and mortality among lupus patients. Established treatment protocols for LN typically include immunosuppressive therapy in combination with glucocorticoids. Although the survival of LN patients has improved over the last 2 decades due to earlier recognition and more 
aggressive intervention, about a quarter of patients continue to progress to end stage renal disease (ESRD). ${ }^{1}$

There is considerable diversity in the definition of 'refractory lupus nephritis' depending on the treatment protocol, physician's subspecialty, and histopathologic findings. Molecular biomarkers further show potential as surrogates to kidney biopsies in predicting renal outcomes and long-term prognosis. ${ }^{2}$ The factor of drug adherence, however, may draw the fine distinction between refractory (or resistant) and relapsing $\mathrm{LN}$, with the latter substantially more common than the former. ${ }^{3}$ Nearly half of patients with proliferative LN who initially achieve a complete response to immunosuppressive therapy will have a relapse or renal flare following cessation or reduction of immunosuppression. Other risk factors for refractoriness include genetics and comorbidities like hypertension, diabetic nephropathy and antiphospholipid antibodies, each of which must be effectively addressed in the overall management of these patients.

Treatment options for refractory $\mathrm{LN}$ include switching or multitargeted therapy with immunosuppressives cyclophosphamide, mycophenolate derivatives and calcineurin inhibitors (cyclosporine A, tacrolimus, and recently voclosporin). ${ }^{4}$ Literature abounds with the use of rituximab in refractory LN including trials exploring the sequential use of rituximab plus cyclophosphamide followed by belimumab. ${ }^{5}$ Other modalities such as extracorporeal treatment (plasma exchange or immunoadsorption) and stem cell transplantation may be tried in special situations. Novel insights of $\mathrm{LN}$ pathogenesis have led to the development of new or re-purposed drugs including obinutuzumab, itolizumab, and iguratimod which can significantly prevent progression to ESRD. Finally, the timely, aggressive and highly individualised management of LN which reinforces strict patient adherence is key to enhanced outcomes.

\section{Learning Objectives}

- Define refractory and/or relapsing LN

- Identify risk factors for refractoriness among LN patients

- Describe the management approach to refractory LN

- Describe advances in drug development for LN

\section{REFERENCES}

1. Weidenbusch M, Bai $Y$, Eder J, et al. Refractory lupus nephritis: a survey. Lupus 2019;28(4):455-64.

2. Parodis I, Tamirou F, Houssiau FA. Prediction of prognosis and renal outcome in lupus nephritis. Lupus Sci Med 2020;7(1).

3. Kronbichler A, Brezina B, Gauckler $P$, et al. Refractory lupus nephritis: When, why and how to treat. Autoimmun Rev 2019;18(5):510-18.

4. Yo JH, Barbour TD, Nicholls K. Management of refractory lupus nephritis: challenges and solutions. Open Access Rheumatol 2019;11:179-88.

5. Zhong $\mathrm{Z}$, Li $\mathrm{H}$, Zhong $\mathrm{H}$, et al. Clinical efficacy and safety of rituximab in lupus nephritis. Drug Des Devel Ther 2019;13:845-56.

\section{\begin{tabular}{l|l}
24 & REFRACTORY LUPUS CYTOPENIAS
\end{tabular}}

Eloisa Bonfá. University of São Paulo Medical School, Brazil

\subsection{6/lupus-2020-la.24}

The aim of this presentation is to provide concise information regarding diagnosis and management of immune mediated hematological manifestations of systemic lupus erythematosus (SLE), specifically autoimmune hemolytic anemia (AIHA), immune mediated thrombocytopenia and immune mediated leukopenia. ${ }^{1-5}$
Studies on hematological manifestations of SLE are scarce and with low quality of evidence, therefore most existent guidelines are supported by hematological data from non-SLE patients. Of note, SLE patients have some peculiarities that must be taken into consideration when choosing the best treatment approach for their hematological conditions.

The key points of each cytopenia are:

a. Autoimmune hemolytic anemia (AHAI) in SLE is usually mediated by warm antibodies (IgG). First-line treatment is glucocorticoid, but there is no consensus to guide second-line therapy. Rituximab and immunosuppressive drugs can be used in refractory cases.

b. Immune thrombocytopenia is an important prognostic indicator of survival in SLE patients. Treatment is usually indicated for patients with a platelet count $<30 \times 10^{9} / \mathrm{mm}^{3}$. First-line therapy remains glucocorticoids (dexamethasone is the hematologists 1st choice), but sustained response after glucocorticoid discontinuation in SLE patients is unlikely to happen. Rituximab and immunosuppressive drugs can be used in refractory cases. Thrombopoietin agonists and splenectomy should be avoided due to high risk of infection and thrombosis

c. Neutropenia may be severe and can lead to morbidity and mortality from increased susceptibility to infection. Severe neutropenia can be successfully treated with granulocyte colony-stimulating factor. Lymphopenia is most often related to drug toxicity and disease activity. Severe lymphopenia may require the use of prophylactic therapy to prevent opportunistic infections. For all cytopenias an extensive evaluation of other causes, particularly drugs and infection should be performed.

Learning Objectives

- Identify and manage immune mediated anemia in lupus

- Discuss diagnosis and treatment options for thrombocytopenia in lupus

- Explain the diagnosis and management of leukopenia in lupus

\section{REFERENCES}

1. Velo-García A, Castro SG, Isenberg DA. The diagnosis and management of the haematologic manifestations of lupus. J Autoimmun 2016;74:139-60.

2. Fanouriakis A, Kostopoulou M, Alunno A, et al. 2019 update of the EULAR recommendations for the management of systemic lupus erythematosus. Ann Rheum Dis 2019;78(6):736-45.

3. Pons-Estel BA, Bonfa E, Soriano ER, et al. First Latin American clinical practice guidelines for the treatment of systemic lupus erythematosus: Latin American Group for the Study of Lupus (GLADEL, Grupo Latino Americano de Estudio de Lupus)-Pan-American League of Associations of Rheumatology (PANLAR). Ann Rheum Dis 2018;77(11):1549-57.

4. Jäger $\mathrm{U}$, Barcellini $\mathrm{W}$, Broome $\mathrm{CM}$, et al. Diagnosis and treatment of autoimmune hemolytic anemia in adults: Recommendations from the First International Consensus Meeting. Blood Rev 2020;41:100648.

5. Neunert CE, Cooper N. Evidence-based management of immune thrombocytopenia: ASH guideline update. Hematology/the Education Program of the American Society of Hematology American Society of Hematology Education Program 2018;2018(1):568-75.

\section{REFRACTORY MUSCULOSKELETAL MANIFESTATIONS}

Bernardo Pons-Estel. Regional Center for Autoimmune and Rheumatic Diseases and the Cardiovascular Institute of Rosario, Argentina

10.1136/lupus-2020-la.25

Musculoskeletal involvement is one of the most common clinical manifestations of systemic lupus erythematosus (SLE), 